\title{
Specific (granulomatous) oral lesions of sarcoidosis: Report of two cases
}

\author{
Joaquim Marcoval ${ }^{1}$, Joan Mañá ${ }^{2}$
}

${ }^{1}$ Servicio de Dermatología, Hospital Universitari de Bellvitge, IDIBELL, Barcelona
${ }^{2}$ Servicio de Medicina Interna, Hospital Universitari de Bellvitge, IDIBELL, Barcelona

Correspondence:

Servicio de Dermatología,

Hospital de Bellvitge

C/ Feixa Llarga s/ $n$

Hospitalet de Llobregat,

08907 Barcelona, Spain

jmarcoval@bellvitgehospital.cat

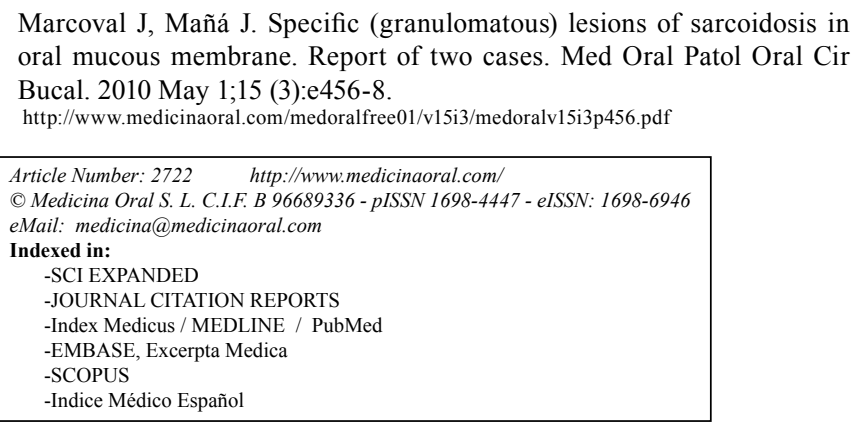

\begin{abstract}
Sarcoidosis is a multisystemic granulomatous disease of unknown etiology that usually presents with bilateral hiliar adenopathies, pulmonary infiltrations, and ocular and cutaneous lesions. The specific cutaneous lesions, or granulomatous, are produced in from $9 \%$ to $37 \%$ of patients with systemic sarcoidosis. Nevertheless, few patients have been described having specific lesions from sarcoidosis in the oral mucous membrane.

We present 2 patients with systemic sarcoidosis who developed specific sarcoidosis lesions in the oral mucous membrane. The first patient presented a plaque on the tongue of a chronic nature associated with facial lupus pernio. The second patient presented a rapidly growing nodular lesion on the lower lip that was in fact the presentation sign of the disease.

Although infrequent, oral lesions may constitute the first sign of systemic sarcoidosis. For this reason the oral mucous membrane needs to be explored when there is suspicion of sarcoidosis, and systemic sarcoidosis must be included in the differential diagnosis of oral granulomatous lesions.
\end{abstract}

Key words: Oral mucous membrane, skin, sarcoidosis. 


\section{Introduction}

Sarcoidosis is a multisystemic granulomatous disease of unknown etiology that usually presents with bilateral hiliar adenopathies, pulmonary infiltrations, and ocular and cutaneous lesions. The diagnosis is established when a clinical and radiological compatible profile is supported by the histological demonstration of non-caseating granulomas in one or more tissues (1).

The specific or granulomatous cutaneous lesions are produced in from $9 \%$ to $37 \%$ of patients with systemic sarcoidosis (2). In addition to being among the organs most frequently affected by the disease, the skin and the oral mucous membrane represent an organ that is easily accessible for the practice of biopsy. The demonstration at the histological level of the presence of sarcoid granulomas of the skin makes it easier to avoid other more potentially aggressive diagnostic procedures $(3,4)$.

However, few patients have been described having specific lesions of sarcoidosis in the oral mucous membrane. We present 2 patients with systemic sarcoidosis who developed specific lesions of sarcoidosis in the oral mucous membrane. In one case this was the presentation sign of the disease.

\section{Clinical Cases}

\section{Case 1}

The patient is a 53-year-old woman presenting facial lesions of some fifteen years' evolution. Of note among her pathological antecedents was pancreatitis in 1986. The patient presented granulomatous-appearing plaques on the nose, the left cheek, and the left eyebrow. Biopsy showed granulomatous dermatitis and the patient was diagnosed with lupus pernio. Systemic sarcoidosis was confirmed with radiology and thoracic CT scan by the presence of bilateral and mediastinic hiliar adenopathies. Upon exploration of the oral mucous membrane the patient presented a slow-growing lesion on the tip of the tongue that had been developing at the same time as the facial lesions (Fig. 1). The plaque was yellowish-brown, discretely infiltrated upon touching, with a smooth surface, disappearance of the filiform papillae, and surface telangiectasias. No other organic sarcoidosis involvements were detected. The patient was treated with oral hydroxicloroquine with temporary improvement of the facial and mucous membrane lesions, which reappeared upon withdrawal from the treatment. During the clinical follow-up the lesions have persisted with slight remission coinciding with the several hydroxicloroquine treatment courses.

\section{Case 2}

Case 2 is a 66-year-old woman, with a history that includes hysterectomy, arterial hypertension, and fibromyalgia. She was referred to the dermatology service for a nodular lesion on the lower lip of three months' evolution (Fig. 2). The lesion was well delimited, elas- tic on touch and covered by semimucous labial tissue that appeared normal. The lesion was biopsied under clinical suspicion of being a neoplastic lesion possibly originating in the minor salivary gland. However, the biopsy revealed that the nodule was made up of sarcoidal non-necrotizing granulomas. Physical exploration at this time showed the presence of papular lesions on the two knees (Fig. 3) and the right elbow that had not previously been detected by the patient. The patient did not present systemic symptomology. Thoracic x-ray produced images compatible with bilateral hiliar adenopathies, and no other organic signs of the disease were

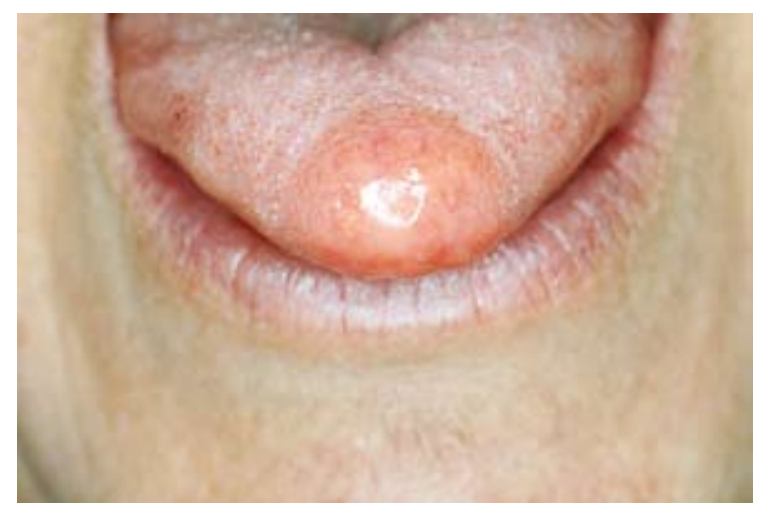

Fig. 1. Case 1. Yellowish-brown plaque on the tip of the tongue with a shiny appearance, with disappearance of filiform papillae.



Fig. 2. Case 2. Nodular lesion on the lower lip.

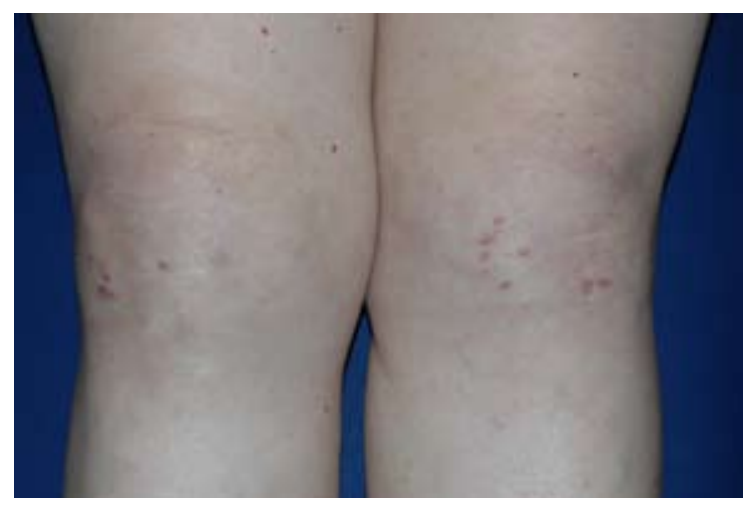

Fig. 3. Case 2. Papular lesions on the knees. 
detected.

\section{Discussion}

Oral lesions are infrequent in sarcoidosis $(5,6)$. A recent review of the English-language literature turned up only 68 well-documented cases of oral sarcoidosis. The average age of the patients was 37-years-old, with a female/ male ratio of 1.5/1. Twenty-one cases involved lesions of the maxillary bone tissue while 47 patients presented lesions in the soft tissue of the oral cavity (6).

In the majority of published cases, sarcoidosis lesions of the oral mucous membrane are unique. The most frequent localization is the jugular mucous membrane, followed by the gums, lips, floor of the mouth, tongue, and palate. In very few cases do the lesions affect more than one localization (6). Clinically, the lesions usually consist of diffuse enlargement at the submucous level or a nodular lesion, firm to the touch, with normal overlying mucous membrane (5). Papulae and superficial ulcerations have also been observed $(5,7)$. No characteristics have been described as specific to any localization. Oral lesions do not normally produce symptomology and they are usually discovered as slow growth enlargements of the mucous membrane (5). Only some cases with pain and dryness of the tongue have been described (5).

Oral manifestations were the first signs of systemic sarcoidosis in 24 of the 68 cases gathered in one review $(6,7)$, and in 2 cases the oral lesions even preceded acute forms of systemic sarcoidosis such as Löfgren syndrome $(8,9)$.

Diverse treatments have been employed for lesions of sarcoidosis in oral mucous membrane. Asymptomatic lesions do not require any treatment, as spontaneous remission is not uncommon (5). The treatment most frequently carried out is surgical extirpation, which is the treatment of choice for singular nodular lesions that are uncomfortable $(5,6)$. The oral administration of corticoids should be considered only for painful or progressive lesions that interfere with the functionality of the oral mucous membrane, making eating or speech difficult $(5,6)$.

The first of our two patients presented with a persistent lesion, of a chronic nature, in association with lesions of lupus pernio on the face. Its yellowish-brown coloration might have suggested its granulomatous nature. Given that the patient was asymptomatic, no specific treatment was carried out. In clinical case 2 the nodular lesion was covered by normal appearing labial mucous membrane; its clinical appearance did not suggest a granulomatous nature and this oriented the clinical approach toward regarding it as a tumoral lesion. The lesion grew in a few weeks' time, then went into remission following biopsy and became associated with papular sarcoidosis of the knees. The similarity between skin and oral mucous membrane suggest that it may be possible to observe, in the oral cavity, the equivalent of the various forms of specific cutaneous lesions of sarcoidosis, from selflimited acute forms that appear at the beginning of the systemic disease to persistent chronic lesions.

Cases like those described in the present article suggest that, for those patients with systemic sarcoidosis, it is advisable to explore the mucous membrane in search of specific lesions, as a means of avoiding more invasive explorations to diagnose the disease. Furthermore, as in clinical case 2, oral lesions may constitute the first sign of systemic sarcoidosis, and systemic sarcoidosis must be borne in mind in the differential diagnosis of oral granulatomous lesions.

\section{References}

1. Hunninghake GW, Costabel U, Ando M, Baughman R, Cordier JF, Du Bois R, et al. ATS/ERS/WASOG statement on sarcoidosis. American Thoracic Society/European Respiratory Society/World Association of Sarcoidosis and other Granulomatous Disorders. Sarcoidosis Vasc Diffuse Lung Dis. 1999;16:149-73.

2. Badrinas F, Morera J, Fité E, Mañá J, Vidal R, Ruiz Manzano J, et al. Sarcoidosis in Catalonia: analysis of 425 cases. Med Clin (Barc). 1989;93:81-7.

3. Mañá J, Marcoval J, Graells J, Salazar A, Peyrí J, Pujol R. Cutaneous involvement in sarcoidosis. Relationship to systemic disease. Arch Dermatol. 1997;133:882-8.

4. Marcoval J, Moreno A, Mañá J. Papular sarcoidosis of the knees: a clue for the diagnosis of erythema nodosum-associated sarcoidosis. J Am Acad Dermatol. 2003;49:75-8.

5. Kasamatsu A, Kanazawa H, Watanabe T, Matsuzaki O. Oral sarcoidosis: report of a case and review of literature. J Oral Maxillofac Surg. 2007;65:1256-9.

6. Suresh L, Radfar L. Oral sarcoidosis: a review of literature. Oral Dis. 2005;11:138-45.

7. Marie I, Proux A, Levesque H, Bony-Rerolle S, Chenal P. Tongue involvement revealing sarcoidosis. QJM. 2008;101:909-11.

8. Jackowski J, Dragisic D, Arnold G, Dirschka T. Primary oral sarcoidosis preceding Lofgren's syndrome. Oral Surg Oral Med Oral Pathol Oral Radiol Endod. 2005;100:183-5.

9. Woodwards RT. Lofgren's syndrome. Br Dent J. 1983;154:143-4. 